\title{
ORIGINAL TABLES.
}

Table showing the Present Valus of £1 per Annum for any number of Years not exceeding 100, at the following rates of Interest.

\begin{tabular}{|c|c|c|c|c|c|c|c|c|}
\hline 袋 & $\begin{array}{c}1 \frac{1}{5} \\
\text { per Cent. }\end{array}$ & $\begin{array}{c}1 \frac{3}{8} \\
\text { per Cent. }\end{array}$ & $\begin{array}{c}1 \frac{5}{8} \\
\text { per Cent. }\end{array}$ & $\begin{array}{c}1 \frac{7}{g} \\
\text { per Cent. }\end{array}$ & $\begin{array}{c}2 \frac{1}{8} \\
\text { per Cent. }\end{array}$ & $\begin{array}{c}2 \frac{3}{8} \\
\text { per Cent. }\end{array}$ & $\begin{array}{c}2 \frac{5}{3} \\
\text { per Cent. }\end{array}$ & $\begin{array}{c}2 \frac{7}{5} \\
\text { per Cent. }\end{array}$ \\
\hline 1 & 88 & 04 & & 116 & $9 / 91$ & 768 & $\cdot 9744$ & $9 / 2$ \\
\hline 2 & & & & 1.9451 & & & & \\
\hline 3 & & & & & & & & \\
\hline 4 & & תי & & & & 33 & & $3 \cdot 7282$ \\
\hline 5 & & & & & & & & $4 \cdot 5960$ \\
\hline 0 & & & $5 \cdot 6$ & $5 \cdot 6251$ & 5.5 & & & $5 \cdot 4397$ \\
\hline 7 & & & & & & & & \\
\hline 8 & & & & $7 \cdot 3651$ & & $7 \cdot 2085$ & & $7 \cdot 0568$ \\
\hline 9 & & & & $8 \cdot 2$ & & & & \\
\hline 10 & 30 & 35 & 4 & $9 \cdot 0416$ & $8 \cdot 9$ & 38 & & 8.5848 \\
\hline 11 & & & & & & & & \\
\hline 12 & & & 10 & & & & & 86 \\
\hline 13 & & & & & & & & \\
\hline 14 & & & & & & & & \\
\hline 15 & & & & & & & & \\
\hline 16 & & & & & & & & \\
\hline 17 & & & & & & & & \\
\hline 10 & & & & & & & & \\
\hline 19 & & & & & & & & \\
\hline 20 & & & & & & & & \\
\hline 21 & & & 7 & & & & & \\
\hline 22 & & & & & & & & \\
\hline 23 & & & & & & & & \\
\hline 24 & & & & & & & & \\
\hline 25 & & & & & & & & \\
\hline 26 & & & & & & & & \\
\hline$a 7$ & & & & & & & & \\
\hline 28 & 63 & & & & & & & \\
\hline $2 y$ & & & & & & & & \\
\hline 30 & & & & & & & & \\
\hline 31 & & & & & & & & \\
\hline 32 & & & & & & & & \\
\hline 33 & & & & & & & & \\
\hline 34 & & & & & & & & \\
\hline 35 & & & & & & & & \\
\hline 36 & & & & & & & & \\
\hline 37 & & & & & & & & \\
\hline 38 & & & & & & & & \\
\hline 32 & & & & & & & & \\
\hline 40 & & & & & & & & \\
\hline 41 & & & & & & & & \\
\hline 42 & & & & & & & & \\
\hline $4:$ & & & & & & & & \\
\hline 44 & & & & & & & & \\
\hline 45 & & & & & & & & \\
\hline 46 & & & & & & & & \\
\hline 47 & & & & & & & & \\
\hline 48 & & & & & & & & \\
\hline 49 & & & & & & & & \\
\hline 50 & & & & & & & & \\
\hline 51 & & & & & & & & \\
\hline 52 & & & & & & & & \\
\hline 5 & 7607 & $7 \cdot 4608$ & $5 \cdot 3495$ & $33-4076$ & $31 \cdot 6$ & $29 \cdot 9697$ & $28 \cdot 4468$ & 7. \\
\hline
\end{tabular}


Table showing the Present Value of $£ 1$ per Annum, \&c. (continued.)

\begin{tabular}{|c|c|c|c|c|c|c|c|c|}
\hline 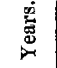 & $\begin{array}{c}1 \frac{1}{8} \\
\text { per Cent. }\end{array}$ & $\begin{array}{c}1 \frac{3}{8} \\
\text { per Cent. }\end{array}$ & $\begin{array}{c}1 \frac{5}{8} \\
\text { per Cent. }\end{array}$ & $\begin{array}{c}1 \frac{7}{8} \\
\text { per Cent. }\end{array}$ & $\begin{array}{c}2 \frac{1}{8} \\
\text { per Cent. }\end{array}$ & $\begin{array}{c}2 \frac{3}{8} \\
\text { per cent. }\end{array}$ & $\begin{array}{c}2 \frac{5}{8} \\
\text { per Cent. }\end{array}$ & $\begin{array}{c}2 \frac{1}{8} \\
\text { per Cent. }\end{array}$ \\
\hline 54 & 972 & 37.9392 & $35 \cdot 7683$ & $33 \cdot 7743$ & $31 \cdot 9402$ & $30 \cdot 2512$ & 286936 & $27 \cdot 2554$ \\
\hline 55 & 40.8477 & & 803 & & 22548 & 30.5262 & 28.9341 & $27 \cdot 4658$ \\
\hline 56 & $41-3822$ & 388765 & 36.5858 & $\mathbf{3 4} \cdot \mathbf{4 8 7 6}$ & 32.5629 & 307948 & $29 \cdot 1684$ & 27.6703 \\
\hline 57 & 41.9107 & $39 \cdot 3356$ & $36 \cdot 9848$ & $34 \cdot 8345$ & $32-8645$ & $31 \cdot 0572$ & $29 \cdot 3968$ & 278690 \\
\hline 58 & $42 \cdot 4333$ & $39 \cdot 7885$ & $37 \cdot 3774$ & $35 \cdot 1750$ & $33 \cdot 1598$ & $31 \cdot 3135$ & $29 \cdot 6193$ & 280622 \\
\hline 59 & $42 \cdot 9502$ & $40-2353$ & $37 \cdot 7638$ & 35.5092 & $33 \cdot 4491$ & $31 \cdot 5639$ & $29 \cdot 8361$ & $28 \cdot 2500$ \\
\hline 60 & 43.4612 & 40.6760 & $38 \cdot 1439$ & 35.8372 & $33 \cdot 7322$ & $31 \cdot 8084$ & $30 \cdot 0473$ & $28 \cdot 4326$ \\
\hline 61 & $43-9666$ & $41 \cdot 1107$ & $38-5180$ & 36.1592 & $34 \cdot 0095$ & $32 \cdot 0473$ & $30 \cdot 2532$ & $28 \cdot 6101$ \\
\hline 62 & $44 \cdot 4664$ & 41.5395 & 388861 & 36.4753 & 34.2811 & $32 \cdot 2807$ & $30 \cdot 4538$ & 287826 \\
\hline 63 & $44 \cdot 9606$ & 41.9626 & $39 \cdot 2483$ & 36.7856 & $34: 5470$ & $32 \cdot 5086$ & 30.6492 & 28.9502 \\
\hline 64 & $45 \cdot 4493$ & 42:3798 & $39 \cdot 6048$ & $37 \cdot 0901$ & $34 \cdot 8073$ & $32 \cdot 7312$ & 308397 & $29 \cdot 1132$ \\
\hline 65 & 45.9326 & 427915 & $39 \cdot 9555$ & $37 \cdot 3891$ & $35 \cdot 0622$ & $32 \cdot 9487$ & $31 \cdot 0253$ & $29 \cdot 2717$ \\
\hline 66 & 46.4105 & $43 \cdot 1975$ & $40 \cdot 3006$ & $37 \cdot 6825$ & $35 \cdot 3118$ & $33 \cdot 1611$ & $31 \cdot 2061$ & 29.4257 \\
\hline 67 & 46.8831 & 4.3 .5980 & $40-6402$ & $37 \cdot 9706$ & 35.5563 & $33 \cdot 3686$ & $31 \cdot 3823$ & 29.5754 \\
\hline 68 & $47 \cdot 3504$ & 43.9931 & 40.9744 & $38-2533$ & $35 \cdot 7956$ & 33.5713 & 31.5540 & $29 \cdot 7209$ \\
\hline 69 & $47 \cdot 8125$ & $44 \cdot 3828$ & $41 \cdot 3032$ & $38 \cdot 5309$ & $36 \cdot 0300$ & $33-7693$ & $3 \mathrm{I} \cdot 7213$ & $29 \cdot 8624$ \\
\hline 70 & $48-2695$ & $44 \cdot 7673$ & $4 I \cdot 6267$ & $38 \cdot 8$ & $36 \div 2595$ & $33 \cdot 9629$ & $31 \cdot 8844$ & $29 \cdot 9999$ \\
\hline 71 & $48 \cdot 7214$ & $45 \cdot 1465$ & $4 I \cdot 9451$ & 39.0708 & $36 * 4842$ & $34 \cdot 1516$ & 132 & $30 \cdot 1335$ \\
\hline 72 & $49 \cdot 1683$ & $45 \cdot 5206$ & $42 \cdot 2584$ & $39 \cdot \mathbf{3 3 3 3}$ & $36-7042$ & $34 \cdot 3361$ & $32 \cdot 1980$ & $30 \cdot 2634$ \\
\hline 73 & $49 \cdot 6102$ & $45 \cdot 8896$ & $42 \cdot 5667$ & 09 & 36.9197 & $34 \cdot 5163$ & $32 \cdot 3489$ & $30 \cdot 3897$ \\
\hline 74 & 50.0471 & $46 \cdot 2536$ & $42 \cdot 8701$ & $39 \cdot 8439$ & $37 \cdot 1306$ & $34 \cdot 6924$ & $32 \cdot 4959$ & 30.5125 \\
\hline 75 & $50 \cdot 4793$ & $46 \cdot 6127$ & 431686 & $40 \cdot 0921$ & $37 \cdot 3372$ & $34 \cdot 8644$ & $32 \cdot 6391$ & $30 \cdot 6318$ \\
\hline 76 & $50 \cdot 9066$ & 46.9669 & $43 \cdot 4623$ & $40 \cdot 3358$ & $37 \cdot 5395$ & 35.0323 & $32 \cdot 7786$ & 307478 \\
\hline 77 & $51 \cdot 3291$ & $47 \cdot 3163$ & 437514 & 40.5751 & $37 \cdot 7376$ & $35 \cdot 1964$ & 32.9146 & $30 \cdot 8606$ \\
\hline 78 & 51.7470 & $47 \cdot 6610$ & 44.0358 & $40 \cdot 8099$ & $37 \cdot 9315$ & $35 \cdot 3567$ & $33 \cdot 0471$ & 30 \\
\hline 79 & $52 \cdot 1602$ & 48.0010 & $44 \cdot 3157$ & $41 \cdot 0404$ & $38-1215$ & 35.5133 & $33 \cdot 1763$ & 31.0767 \\
\hline 80 & $52 \cdot 5688$ & $48 \cdot 3363$ & $44-5911$ & $41 \cdot 2666$ & $38: 3074$ & 62 & 21 & $31-1803$ \\
\hline 81 & $52 \cdot 9729$ & 48.6672 & 44.8620 & $41 \cdot 4887$ & $38 \cdot 4895$ & 35.8156 & $33 \cdot 4247$ & $31 \cdot 2810$ \\
\hline 82 & $53 \cdot 3725$ & 489935 & & $41 \cdot 7067$ & $38 \cdot 6678$ & 15 & 33.5441 & $31 \cdot 3788$ \\
\hline 83 & $53 \cdot 7676$ & $49 \cdot 3154$ & 45.3911 & 41.9207 & $38-8424$ & $36 \cdot 1040$ & 336605 & 31.4739 \\
\hline 84 & $54 \cdot 1583$ & 49.6330 & 45.6493 & $42^{\circ}$ & $39-0134$ & $36 \cdot 2432$ & 40 & $31 \cdot 5664$ \\
\hline 85 & $54 \cdot 5447$ & $49 \cdot 9462$ & 45.9034 & $42-3369$ & $39 \cdot 1808$ & 36.3792 & 33.8845 & $31 \cdot 6563$ \\
\hline 86 & $54 \cdot 9268$ & $50 \cdot 2552$ & $46 \cdot 1584$ & $42 \cdot 5393$ & $39-344 \tau$ & 36.5121 & 222 & $3 \mathrm{~J} \cdot 7436$ \\
\hline 87 & $55 \cdot 3046$ & 50.5600 & $46 \cdot 3994$ & $42 \cdot 7380$ & 39.50 .52 & $36 \cdot 6418$ & 34.0972 & 31.3286 \\
\hline 88 & $55 \cdot 6783$ & 50.8607 & 466415 & $42 \cdot 9330$ & $39 \cdot 6624$ & $36 \cdot 7686$ & $34 \cdot 1994$ & 31.9111 \\
\hline 89 & 56.0477 & 51.1572 & 468797 & $43 \cdot 1244$ & 39.8163 & 36.8924 & $34-2991$ & $31 \cdot 9913$ \\
\hline 90 & $56 \cdot 4131$ & 51.4498 & $47 \cdot 1141$ & $43 * 3123$ & 39.9670 & 37.0133 & 34.3961 & 32.0693 \\
\hline 91 & 56.7744 & $51 \cdot 7384$ & $47 \div 9447$ & 434967 & $40-1146$ & $37 \cdot 1314$ & $34^{\circ} 4908$ & $32 \cdot 1452$ \\
\hline 92 & $57 \cdot 1317$ & $52 \cdot 0231$ & 47.5717 & 43.6778 & 40.2591 & 37.2468 & 34.5829 & 322189 \\
\hline 93 & 574850 & $52 \cdot 3039$ & 477950 & 438555 & 40.4005 & 37.3595 & $34 \cdot 6728$ & $32-2905$ \\
\hline 94 & $57 \cdot 8344$ & 52.5809 & 48.0148 & 44.0299 & 40.5391 & 37.4696 & $34 \cdot 7603$ & $32 \cdot 3601$ \\
\hline 95 & $58 \cdot 1799$ & $52: 8542$ & $48 \cdot 2310$ & $44 \cdot 2012$ & & $37 \cdot 5772$ & 34.8456 & $32 \cdot 4278$ \\
\hline 96 & $58 \cdot 5215$ & $53 \cdot 1237$ & $48 \cdot 4438$ & $44-3692$ & 40.8076 & 37.6822 & 34.9287 & $32 \cdot 4936$ \\
\hline 97 & 58.8594 & $53 \cdot 3896$ & $48 \cdot 6532$ & $44: 5342$ & $40 \cdot 9377$ & 377848 & 35.0097 & 32.5576 \\
\hline 98 & $59 \cdot 1934$ & 53.6519 & 48.8592 & 44.6962 & 41.0650 & 37.8851 & 35.0886 & 326198 \\
\hline 99 & $59 \cdot 5238$ & 53.9106 & $49 \cdot 0620$ & 44.8551 & $41 \cdot 1897$ & 37.9830 & $35 \cdot 1655$ & $32 \cdot 6802$ \\
\hline 100 & $59 \cdot 8505$ & $54 \cdot 1658$ & $49-2615$ & 450112 & $41 \cdot 3119$ & 380786 & 35.2405 & 32.7390 \\
\hline$p^{p}$ & 88.8888 & $72 \cdot 7272$ & 61.5384 & $53 \mathbf{3 8 3 3}$ & $47 \cdot 0588$ & 421052 & $38 \cdot 0952$ & $34 \cdot 7826$ \\
\hline
\end{tabular}

Our readers are indebted to Mr. PeTER Hardy for this Table.

END OF VOL. IV. 